\title{
Ultrahigh brightness electron beams by plasma-based injectors for driving all-optical free-electron lasers
}

\author{
V. Petrillo, ${ }^{1,2}$ L. Serafini, ${ }^{1}$ and P. Tomassini ${ }^{1,3}$ \\ ${ }^{1}$ INFN-Milan, Via Celoria 16, 20133, Milano, Italy \\ ${ }^{2}$ Università degli Studi di Milano, Dipartimento di Fisica, Via Celoria 16, 20133, Milano, Italy \\ ${ }^{3}$ CNR-ILIL, Via G. Moruzzi, 1, 56124, Pisa, Italy \\ (Received 27 March 2008; published 29 July 2008)
}

\begin{abstract}
We studied the generation of low emittance high current monoenergetic beams from plasma waves driven by ultrashort laser pulses, in view of achieving beam brightness of interest for free-electron laser (FEL) applications. The aim is to show the feasibility of generating $\mathrm{nC}$ charged beams carrying peak currents much higher than those attainable with photoinjectors, together with comparable emittances and energy spread, compatibly with typical FEL requirements. We identified two regimes: the first is based on a laser wakefield acceleration plasma driving scheme on a gas jet modulated in areas of different densities with sharp density gradients. The second regime is the so-called bubble regime, leaving a full electron-free zone behind the driving laser pulse: with this technique peak currents in excess of $100 \mathrm{kA}$ are achievable. We have focused on the first regime, because it seems more promising in terms of beam emittance. Simulations carried out using VORPAL show, in fact, that in the first regime, using a properly density modulated gas jet, it is possible to generate beams at energies of about $30 \mathrm{MeV}$ with peak currents of $20 \mathrm{kA}$, slice transverse emittances as low as $0.3 \mathrm{~mm} \mathrm{mrad}$, and energy spread around $0.4 \%$. These beams break the barrier of $10^{18} \mathrm{~A} /(\mathrm{mm} \mathrm{mrad})^{2}$ in brightness, a value definitely above the ultimate performances of photoinjectors, therefore opening a new range of opportunities for FEL applications. A few examples of FELs driven by such kind of beams injected into laser undulators are finally shown. The system constituted by the electron beam under the effect of the electromagnetic undulator has been named AOFEL (for all optical free-electron laser).
\end{abstract}

DOI: 10.1103/PhysRevSTAB.11.070703

PACS numbers: 52.59. $-\mathrm{f}$

\section{INTRODUCTION}

Recently a few authors proposed to use plasma injectors as an electron beam driver for the self-amplified spontaneous emission (SASE) x-ray free-electron laser (FEL): the aim is to design and build compact FELs, taking advantage from the capability of plasma accelerators to produce $\mathrm{GeV}$ beams on mm-scale lengths, to be compared to $\mathrm{km}$ based $\mathrm{rf}$ linacs. Gruner et al. [1] proposed the use of a plasma injector operated in the bubble regime to generate an electron beam with unprecedented high brightness at an energy of $1 \mathrm{GeV}$, carrying a beam peak current in excess of $100 \mathrm{kA}$ with rather good normalized emittance ( $1 \mathrm{~mm} \mathrm{rad})$ and low energy spread $(0.1 \%)$. This ultrahigh brightness beam is an ideal candidate to drive FELs with radiation wavelength down in the angstrom range: in Ref. [1] the transport and matching of this beam into a magnetostatic undulator is analyzed by means of numerical simulations showing the strong blowup that the beam undergoes along the transport due to its very intense space charge field. The possibility to preserve the beam quality throughout the undulator, as required to maintain the FEL exponential gain regime, is somewhat controversial, mainly because the space charge effects in the longitudinal and in the transverse planes are not negligible on the scale of the FEL gain length.
In this paper we present a different approach, which has in common the goal to use a plasma injector to drive a compact SASE FEL, but differing in the type of regime used in the plasma channel to generate the electron beam and in the energy of the beam itself, which is in the range of a few tens of $\mathrm{MeV}$ instead of a few $\mathrm{GeV}$. To this purpose the use of an electromagnetic undulator, i.e., a counterpropagating laser pulse of proper wavelength, is foreseen, as recently proposed $[2,3]$. The technique of controlling the breaking of the plasma wave by tapering the plasma density in the gas-jet is applied, as proposed in [4-7]: we believe this technique is suitable to produce higher quality beams than the bubble regime, though with lower beam currents (tens of kA instead of higher than $100 \mathrm{kA}$ ). In addition, the use of an electromagnetic (e.m.) undulator allows one to conceive an ultracompact device, a few $\mathrm{mm}$ of total length, compared to meters of the scheme using $\mathrm{GeV}$ beams. If the e.m. laser pulse is of the same wavelength of the laser pulse driving the plasma wave (or close to it), the electron beam does not even need to be extracted from the plasma channel, and the FEL can be driven in absence of space charge effects at all. Indeed the e.m. laser pulse can be injected into the plasma channel, so that the FEL interaction can take place inside the plasma. If the e.m undulator is made out of a $\mathrm{CO}_{2}$ laser pulse, while the plasma density is larger than $10^{19} \mathrm{~cm}^{-3}$, it cannot propa- 


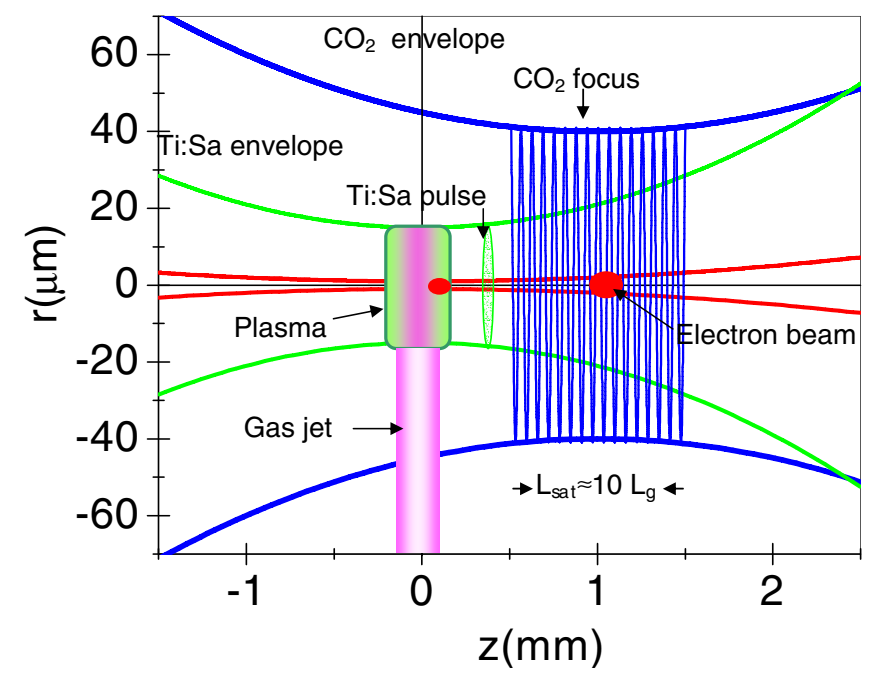

FIG. 1. (Color) Layout of the experiment for the case of the $\mathrm{CO}_{2}$ electromagnetic undulator for which the plasma density is overcritical (see text for details).

gate through the plasma because it is undercritical: in this case the typical layout is presented in Fig. 1.

\section{E-BEAM GENERATION VIA LWFA}

Laser wakefield acceleration (LWFA) of e-beams [6-8] has been proved to be able to produce high energetic (up to the $\mathrm{GeV}$ scale) quasimonochromatic electron beams $[9,10]$ by using ultrashort (tens of femtosecond long) laser pulses and a plasma as accelerating medium. In LWFA the longitudinal ponderomotive force of the laser pulse excites a plasma wave whose longitudinal (i.e. accelerating) electric field can exceed thousands times that of rf guns. Both experimental and modeling/numerical sides are under active investigation, with emphasis on beam quality, i.e., monochromaticity and low emittance.

A critical issue for achieving high-quality e-beams is represented by a good control in particle injection into the plasma wave, whose phase speed equals the group velocity of the laser pulse. Several schemes have been proposed and investigated so far, including injection of externally preaccelerated e-beams, injection of short burst of newborn electrons produced via tunneling ionization, transverse wave breaking in density downramp [11], transverse injection in the bubble regime [12], and longitudinal wave breaking in density downramp [4-7].

In this paper we will show results of numerical simulation in the nonlinear LWFA regime with longitudinal injection after density downramp. In this scheme the electrons of the crest of the waves are longitudinally injected in the fast running wakefield by a partial break of the wave induced by a sudden change in its phase speed at the transition [4]. If the laser pulse waist $w_{0}$ is much larger than the wave wavelength $\lambda_{p} \cong \sqrt{1.1 \times 10^{21} / n_{e}^{2}}, n_{e}$ being the plasma electron density in $\mathrm{cm}^{-3}$, the transverse dynamics is negligible at the transition and an electron bunch with an extremely low transverse emittance is created and injected in the acceleration region of the wave $[5,6]$.

An analogous phenomenon, but in the plasma Wakefield regime, has been described in Ref. [7].

We searched for parameters giving rise to electron bunches suitable for driving the laser-based FEL source. This means that the e-bunches must have a high current but do not need to be characterized by overall low emittance and energy spread. They should contain slices with very low slice emittance and very low slice energy spread, instead. We expect space charge effects to occur over a beam-plasma wavelength scale, which is given by $\lambda_{\perp \text { bp }}=$ $2 \pi \gamma R \sqrt{\frac{2 \gamma I_{0}}{I}}, R$ being the beam radius, $I$ its current, $\gamma$ the mean Lorentz factor of the electrons, and $I_{0}=17 \mathrm{kA}$ the Alfven current. For our typical beam this length is of the order of a cm, much longer than the expected saturation length in the e.m. undulator (which is of the order of $1 \mathrm{~mm}$ or a fraction of it). On the other hand, this length is typically of the order of $\mathrm{km}$ for an x-ray FEL driven by a $\mathrm{GeV}$ rf electron linac (again much longer than saturation length in a magnetostatic undulator). This implies that we should not expect serious emittance degradation due to transverse space charge along the e.m. undulator, as can be seen from Fig. 2, which presents a simulation made with ASTRA [13] for a typical all optical free-electron laser (AOFEL) beam initially focused to $1 \mu \mathrm{m}$ rms spot size and then freely propagating in a $1.5 \mathrm{~mm}$ long drift. The rms beam envelope $\left\langle\sigma_{x}\right\rangle$ (left scale) and the rms normalized emittance $\varepsilon_{n}$ (right scale) are shown on the range of interest along the coordinate $z$, both with [curves (a)] and without [curves (b)] the effects of the energy spread for $I=$ $20 \mathrm{kA}, \varepsilon_{n}=0.3 \mathrm{mmmrad}$, and $\gamma=60$. The predicted gain length is about $50 \mu \mathrm{m}$, so that the beam evolution is followed for about 30 gain lengths. In $1.5 \mathrm{~mm}$ of drift the

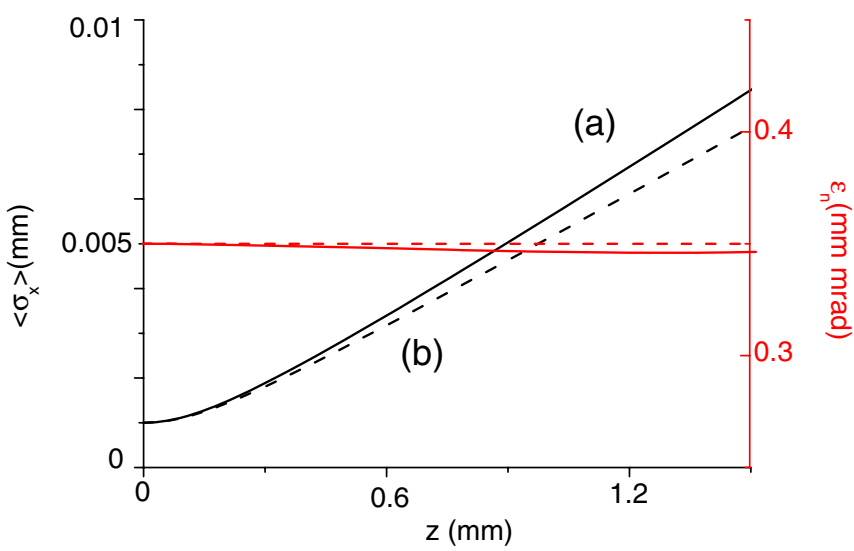

FIG. 2. (Color) Envelope $\left\langle\sigma_{x}\right\rangle$ and transverse normalized emittance $\varepsilon_{n}$ versus $z$ as given by ASTRA in vacuum for a typical AOFEL beam with $I=20 \mathrm{kA}, \varepsilon_{n}(z=0)=0.3 \mathrm{~mm} \mathrm{mrad}$, $\left\langle\sigma_{x}\right\rangle(z=0)=1 \mu \mathrm{m}$, and $\gamma=60$. 


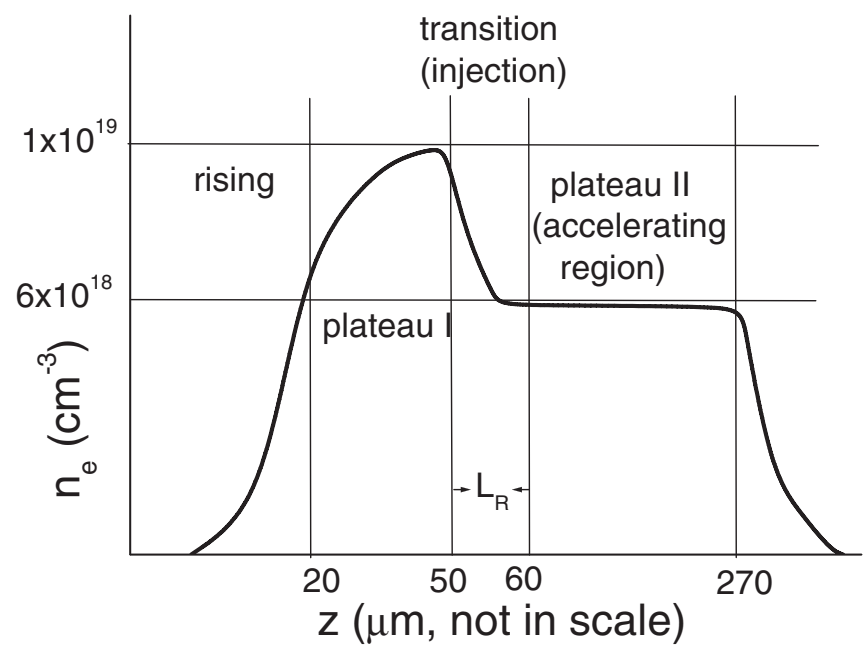

FIG. 3. Background plasma density useful for e-beam injection and acceleration.

a)

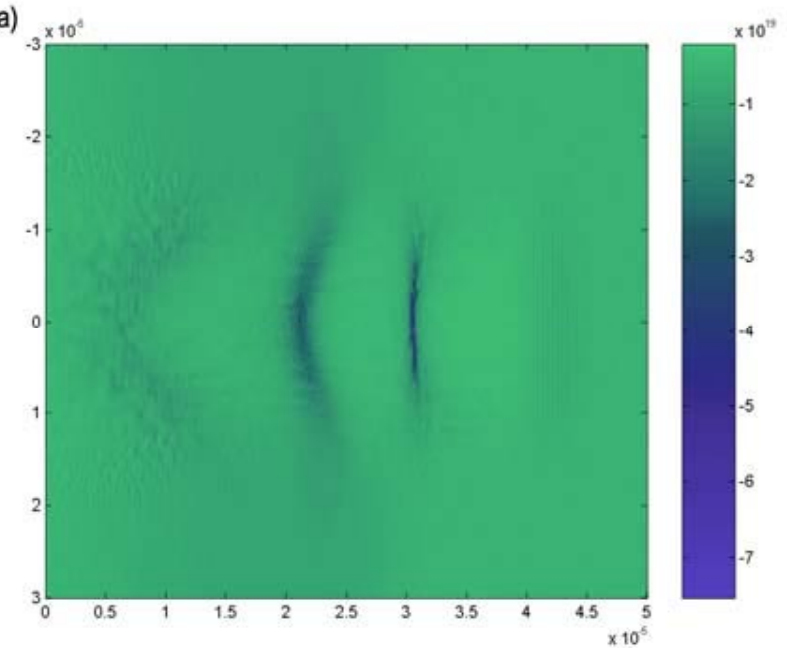

c)

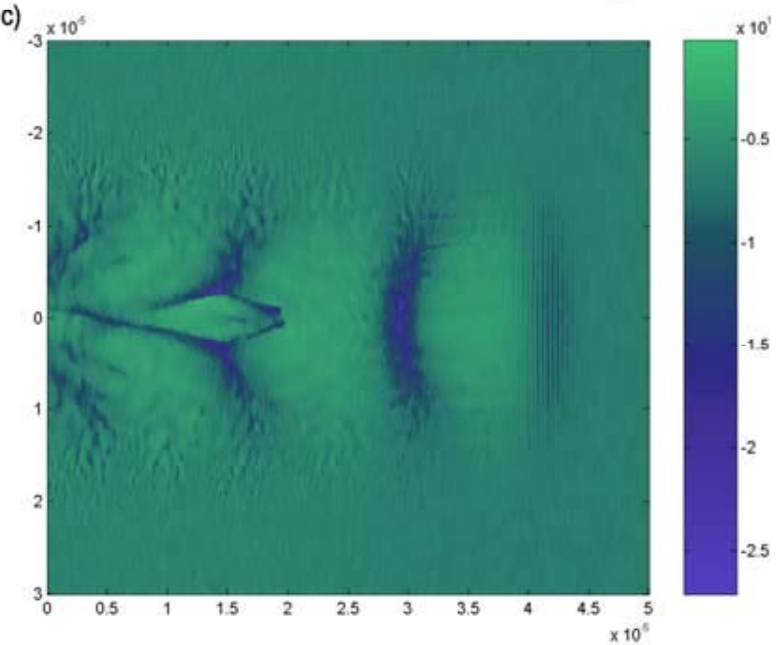

emittance does not change significantly, while the envelope evolves as if the space charge is absent.

Clearly, there may be effects of energy spread increase due to longitudinal and transverse space charge fields, including retarded effects at plasma-vacuum interface, which should be modeled by a self-consistent simulation taking the beam from the plasma to the vacuum region and all the way through the e.m. undulator. Simulations were performed with the fully self-consistent particle-in-cell (PIC) code VORPAL in the 2.5D (3D in the fields, 2D in the coordinates) configuration. The parameters used in the calculations are presented in Table I. VORPAL [14] is a multipurpose code and in the present work it worked in the self-consistent PIC configuration with dynamics in a moving window box moving at the light speed.

The initial plasma density profile (see Fig. 3) is composed of a smooth rising edge (laser coming from the left) and a first plateau of density $n_{01}$, with an appropriate phase of the plasma wave excited in the second plateau (accel-

b)

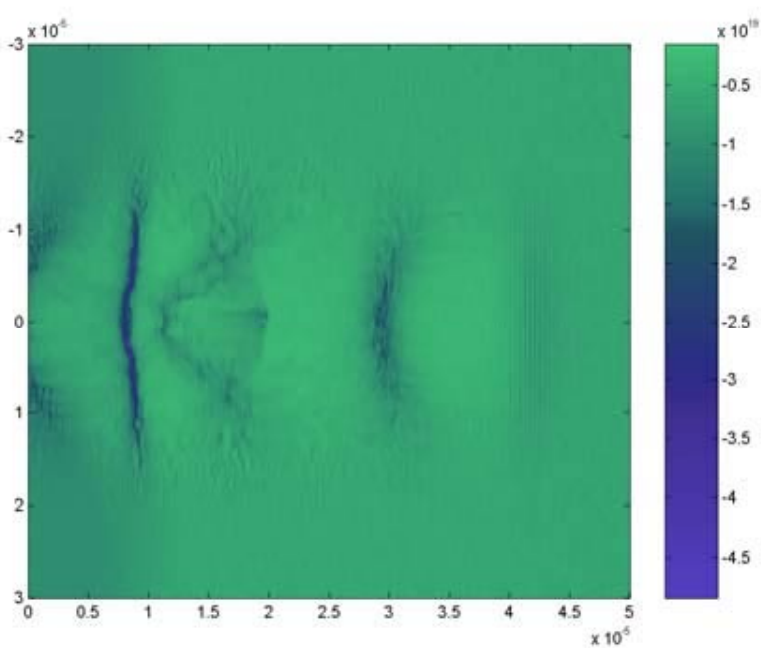

d)

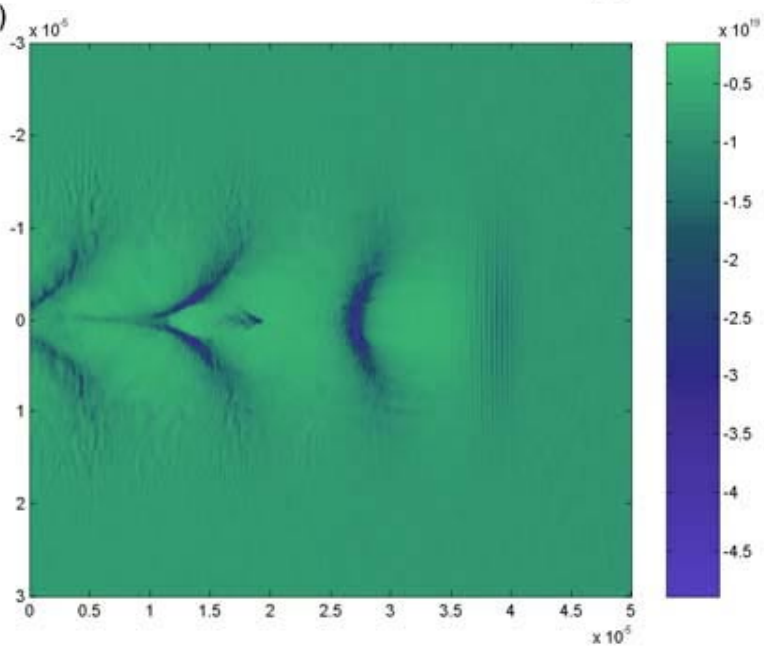

FIG. 4. (Color) Snapshot of the electronic density when the laser pulse is in the first plateau (a), the laser pulse is crossing the transition (b), the laser pulse is just in the accelerating region (c), and the laser pulse has propagated for about $200 \mu \mathrm{m}$ in the accelerating region $(\mathrm{d})$. 
TABLE I. Values of the characteristics of laser and plasma.

\begin{tabular}{cccccccc}
\hline \hline & \multicolumn{5}{c}{ Laser } & \multicolumn{4}{c}{ Plasma } \\
\hline Energy & Waist & Intensity & Duration & $n_{01}$ & $L_{R}$ & $n_{02}$ & $\lambda p$ \\
\hline$(\mathrm{J})$ & $(\mu \mathrm{m})$ & $\left(\mathrm{W} / \mathrm{cm}^{2}\right)$ & $(\mathrm{fs})$ & $\left(1 / \mathrm{cm}^{3}\right)$ & $(\mu \mathrm{m})$ & $1 / \mathrm{cm}^{3}$ & $(\mu \mathrm{m})$ \\
2 & 20 & $7 \times 10^{18}$ & 20 & $1 \times 10^{19}$ & 10 & $0,6 \times 10^{19}$ & 13 \\
\hline \hline
\end{tabular}

erating region). Simulations of the injection-acceleration process were performed in a moving window of longitudinal and transverse size of 50 and $60 \mu \mathrm{m}$, respectively, sampled in an $800 \times 120$ box with 20 macroparticles/ cell, corresponding to a longitudinal and transverse resolution of $\lambda_{0} / 14$ and $\lambda_{0} / 2$, respectively.

In Fig. 4 some snapshots of the electronic density are reported. In Fig. 4(a) the laser pulse (coming to the lefthand side) is crossing the transition and the plasma wave in the first plateau is experiencing nonlinear steepening. In Fig. 4(b) the laser pulse is well inside the second plateau (the accelerating region) and the second crest of the plasma wave has partially broken. The electrons injected in the wave are suddenly accelerated and focused by the longitudinal and transverse forces of the wakefield [Fig. 4(c)]. After an acceleration length of about $200 \mu \mathrm{m}$ the electron beam is still focused [Fig. 4(d)] and gets a maximum energy of about $28 \mathrm{MeV}$.

The final parameters of the initial plasma density have been obtained by an optimization procedure in the parameters double-plateau profile, i.e., the second plateau density, the transition scale length, and the amplitude of the transition. The first parameter controls the plasma wavelength and maximum accelerating gradient in the acceleration region and must be tuned according to the e-beam length and charge. The transition scale length controls the charge and length of the beam and must be as small as possible in order to ensure the trapping of the largest number of particles [4], while a tuning in the amplitude of the transition is necessary for selecting the appropriate phase of the particles in the Langmuir wave.

\section{FEL SIMULATIONS}

The electron bunch produced by VORPAL has been analyzed for finding the slices characterized by the highest brightness for producing FEL radiation [15].

In Fig. 5 the beam current along the beam coordinate $s$ is presented, while Fig. 6 shows the longitudinal phase space. The part shaded, delimited by $z=1.1 \mu \mathrm{m}$ and $1.59 \mu \mathrm{m}$, satisfies both conditions of large current and low energy spread. The average values for this part of the bunch are $\gamma=55.2, I=20 \mathrm{kA}$, and $\left\langle\sigma_{x}\right\rangle=0.5 \mu \mathrm{m}$, and, to be conservative, we have assumed the pessimistic estimates $\delta \gamma / \gamma=1.2 \times 10^{-2}$ and $\varepsilon_{n}=0.3 \mu \mathrm{m}$. We suppose to let the beam defocus up to $\left\langle\sigma_{x}\right\rangle=5 \mu \mathrm{m}$ and subject it to the field of a $\mathrm{CO}_{2}$ laser with wavelength $\lambda_{L}=10 \mu \mathrm{m}$ and intensity $a_{L}=0.8$. For these values the radiation wave- length is $\lambda_{R}=1.35 \mathrm{~nm}$, the FEL parameter $\rho=$ $3 \times 10^{-3}$, the cooperation length $L_{c}=\frac{\lambda_{R}}{4 \pi \sqrt{3} \rho}=$ $0.14 \mu \mathrm{m}$. This last is the length covered by the radiation in the beam reference frame during one gain length. The radiation permits to all the electrons inside this length to enter in correlation and to emit coherently. When the beam length $L_{b}$ is shorter or of the order of $2 \pi L_{c}$, all the electrons in the beam emit coherently, giving rise to a

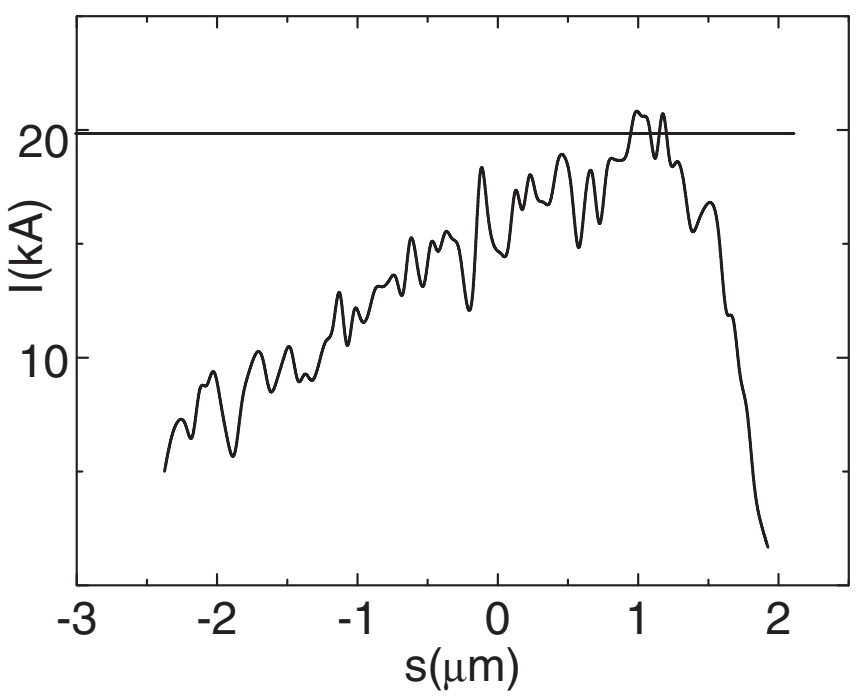

FIG. 5. Current $I$ versus the coordinate along the bunch $s$ in $\mu \mathrm{m}$.

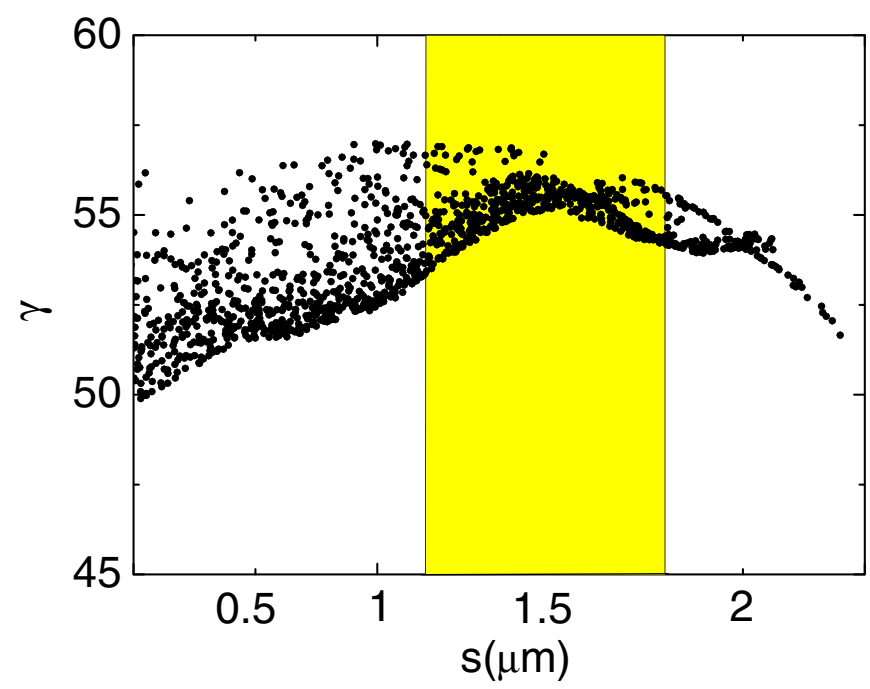

FIG. 6. (Color) Longitudinal phase space $\gamma$ vs $s(\mu \mathrm{m})$. 

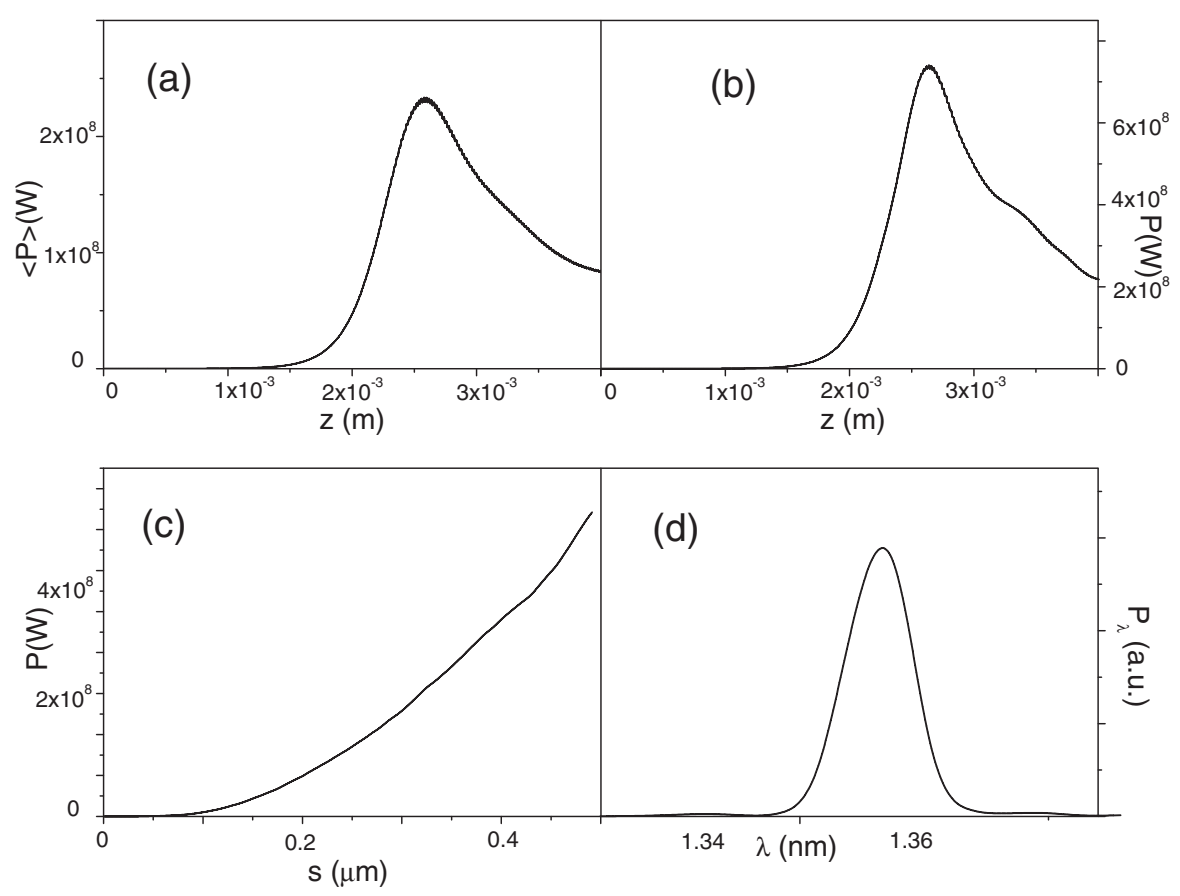

FIG. 7. (a) average power $P(\mathrm{~W})$ vs the coordinate along the undulator $z(\mathrm{~m})$; (b) peak power along $z$; (c) power profile along the bunch coordinate $s(\mu \mathrm{m})$ at $z=2.5 \mathrm{~mm}$; (d) power spectrum at $z=2.5 \mathrm{~mm}$.

superradiant single spike pulse. In our case, $L_{b} / 2 \pi L_{c}=$ 0.566 and the condition $L_{b} / 2 \pi L_{c}<1$ for a clean single spike production turns out to be satisfied.

We want to point out that the dynamics of the beam in the transition between the plasma and the interaction region with the laser has not been so far simulated consistently, so that the sequence of our numerical calculations cannot be considered a complete start-to-end simulation. The effective parameters of the beam at the entrance of the e.m. undulator could be worse than those used. However, estimates of the emittance and of the energy spread made by importing the plasma fields given by VORPAL] in RETAR [13] are in progress [16].

The laser beam interaction has been simulated with the code GENESIS 1.3 [17], which tracks the particles in a static wiggler. The code GENESIS 1.3 is widely used in the FEL predictions and integrates the FEL equations based on the slowly varying envelope approximation [15]. By exploiting the equivalence between static and electromagnetic undulators [3], we have inserted the mean parameters of the bunch radiating slice into GENESIS 1.3, obtaining the results presented in Fig. 7. Window (a) shows the average power $P$ vs the coordinate along the undulator $z$, while window (b) presents the peak power vs $z$. In Fig. 7(c) there is the shape of the power vs $s$ and in (d) the spectrum of the x-rays pulse at $z=2.5 \mathrm{~mm}$. The peak power value is $0.75 \mathrm{GW}$ and the structure of the power on the beam is that typical of superradiance.

In fact, even if the initial radiation field and bunching present a sequence of several random spikes, during the propagation in the wiggler they clean up assuming a smooth shape on the bunch [see (c)], with a neat, thin spectrum. The saturation occurs at $2.5 \mathrm{~mm}$, the subsequent decrease of average and peak power being due to the slippage of the radiation outside the simulated slices.

The effect of $\gamma, \delta \gamma / \gamma$, and $I$ profiles has been then analyzed. In Fig. 8, $\gamma$ and $\delta \gamma / \gamma$ profiles deduced from the effective particle packet are presented, while Fig. 9 shows the results obtained taking the profiles into account.

A situation substantially similar to the preceding one is shown by the graphs, with a slight reduction of the satura-

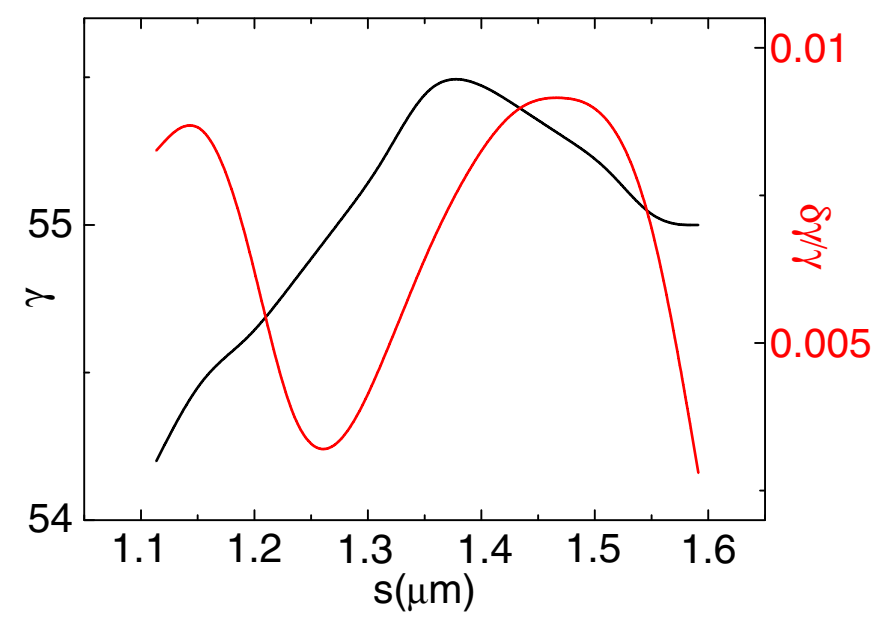

FIG. 8. (Color) Profile of $\gamma$ and $\delta \gamma / \gamma$ vs $s$ deduced by the VORPAL bunch. 

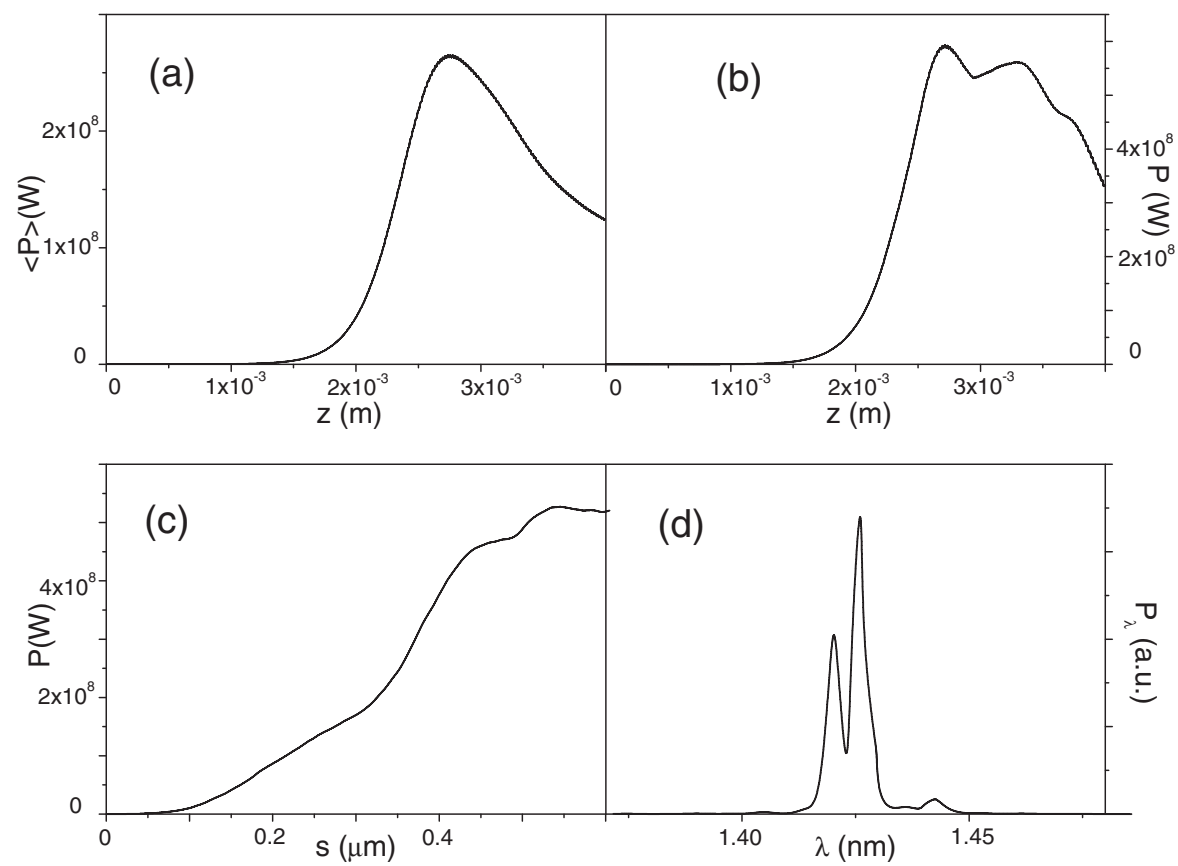

FIG. 9. (a) average power $P(\mathrm{~W})$ vs the coordinate along the undulator $z(\mathrm{~m})$; (b) peak power along $z$; (c) power profile along the bunch coordinate $s(\mu \mathrm{m})$ at $z=2.5 \mathrm{~mm}$; (d) power spectrum at $z=2.5 \mathrm{~mm}$.

tion power and an increase of about $15 \%$ in the saturation length, that turns out to be slightly less than $3 \mathrm{~mm}$.

A further step has been the use of the original files by VORPAL, the results being presented in Fig. 10. The superradiant behavior maintains, and there is the occurrence of a first large peak of radiation, high in power and temporary very thin, with a FWHM width of about $0.1 \mu \mathrm{m}$, corresponding to about 300 attosec, and with a maximum value of $200 \mathrm{MW}$. The transverse coherence is only partial, with the development of a dozen of peaks, while the temporal coherence is strong as can be seen from the pulse in the frequency domain. The same bunch, but without the defo-
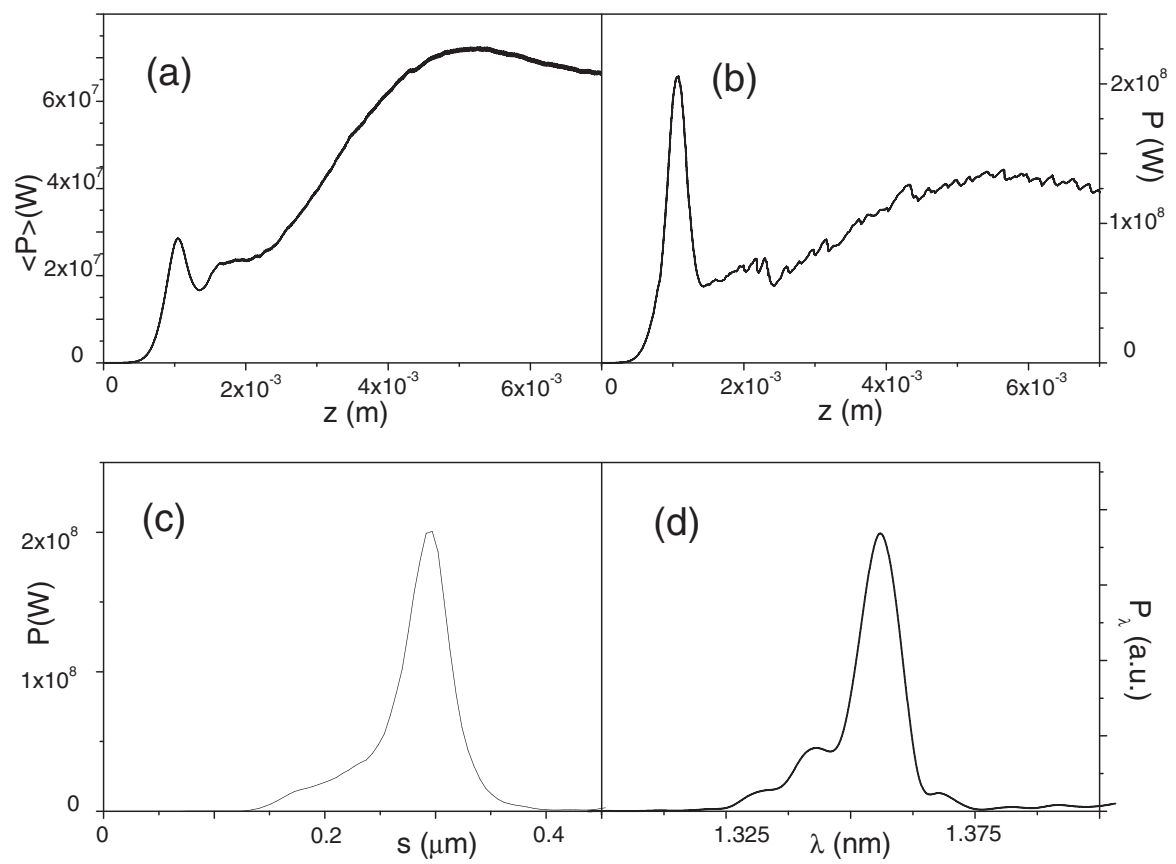

FIG. 10. (a) average power $P(\mathrm{~W})$ vs the coordinate along the undulator $z(\mathrm{~m})$; (b) peak power along $z$; (c) power profile along the bunch coordinate $s(\mu \mathrm{m})$ at $z=2.5 \mathrm{~mm}$; (d) power spectrum at $z=2.5 \mathrm{~mm}$. 
TABLE II. Radiation parameters.

\begin{tabular}{lcccccc}
\hline \hline & $P_{\max }(\mathrm{W})$ & $E(\mu \mathrm{J})$ & $L_{R}(\mu \mathrm{m})$ & $L_{\text {sat }}(\mathrm{mm})$ & $\lambda_{R}(\mathrm{~nm})$ & $\delta \lambda_{R} / \lambda_{R}$ \\
\hline Case 1 & $7.5 \times 10^{8}$ & 0.4 & 0.5 & 2.5 & 1.36 & $0.82 \%$ \\
\hline Case 2 & $6 \times 10^{8}$ & 0.5 & 0.6 & 2.7 & 1.43 & $0.16 \%$ \\
\hline Case 3 & & & & & & \\
First peak & $2 \times 10^{8}$ & 0.05 & 0.05 & 1 & 1.35 & $0.81 \%$ \\
Saturation & $1.5 \times 10^{8}$ & 0.12 & 0.5 & 4.5 & & \\
\hline \hline
\end{tabular}

cusing phase, has been simulated in the field of a laser with wavelength $\lambda_{L}=1 \mu \mathrm{m}$. We observe the production of a clean superradiant peak of width less than $0.05 \mu \mathrm{m}$ (100 attosec), of tens MW and with a clean spectrum.

The results of the FEL simulation have been summarized in Table II, where the peak power $P_{\max }$, the energy extracted $E$, the pulse length $L_{R}$, the saturation length $L_{\text {sat }}$, the radiation wavelength $\lambda_{R}$, and the spectral width $\delta \lambda_{R} / \lambda_{R}$ at saturation have been reported.

Case 1 refers to the calculation presented in Fig. 7, where the average beam parameters have been assigned. Case 2, shown in Fig. 9, refers to the calculation made with an ideal beam with profiles, while in case 3 (Fig. 10) the beam produced by VORPAL has been imported in GENESIS 1.3.

\section{CONCLUSIONS}

In this paper we propose the possibility of using a LWFA plasma driving scheme on a gas-jet modulated in areas of different densities with sharp density gradients to generate a monoenergetic, high current ( $>20 \mathrm{KA}$ ) electron beam.

This bunch of electrons, characterized by the presence of low emittance ( $<0.5 \mathrm{~mm} \mathrm{mrad}$ ), low energy spread slices, when interacting with a counterpropagating $\mathrm{CO}_{2}$ laser pulse, radiates a single spike, highly coherent, ultrashort $(<1 \mathrm{fsec}) \mathrm{x}$-ray pulse via excitation of a SASE superradiant FEL instability. The peak power achieved at $1.2 \mathrm{~nm}$ is of the order of $200 \mathrm{MW}$.

Taking an electromagnetic undulator at $1 \mu \mathrm{m}$ wavelength, the electron beam can drive a $1 \AA$ FEL, with similar performances.

Although the presented analysis is based on threedimensional simulations, effects due to alignment errors, spatial and temporal jitters, as well as realistic laser pulse profiles for the e.m. undulator and consistent exit from the plasma, that have not been yet considered, must be thoroughly investigated in order to assess the experimental feasibility of such a new FEL scheme. Nevertheless, we believe that our analysis, although very preliminary and missing a full self-consistent start-to-end simulation, shows a potential for the future development of an ultracompact source of coherent $\mathrm{x}$ rays at brilliance levels comparable to those typical of fourth generation light sources. Further work is in progress with more consistent simulations able to describe the exit of the beam from the plasma and the transport through the e.m. undulator, in order to address the problem of performing a real start-toend simulation from the plasma to the FEL radiation production.

[1] F. Grüner, S. Becker, U. Schramm, T. Eichner, M. Fuchs, R. Weingartner, D. Habs, J. Meyer-ter-Vehn, M. Geissler, M. Ferrario, L. Serafini, B. Van der Geer, H. Backe, W. Lauth, and S. Reiche, Appl. Phys. B 86, 431 (2007).

[2] A. Bacci, M. Ferrario, C. Maroli, V. Petrillo, and L. Serafini, Phys. Rev. ST Accel. Beams 9, 060704 (2006).

[3] A. Bacci, C. Maroli, V. Petrillo, A. R. Rossi, L. Serafini, and P. Tomassini, Nucl. Instrum. Methods Phys. Res., Sect. A 587, 388 (2008).

[4] S. Bulanov, N. Naumova, F. Pegoraro, and J. Sakai, Phys. Rev. E 58, R5257 (1998).

[5] P. Tomassini, M. Galimberti, A. Giulietti, D. Giulietti, L. A. Gizzi, L. Labate, and F. Pegoraro, Phys. Rev. ST Accel. Beams 6, 121301 (2003).

[6] P. Tomassini, M. Galimberti, A. Giulietti, D. Giulietti, L. A. Gizzi, L. Labate, and F. Pegoraro, Laser Part. Beams 22, 423 (2004).

[7] H. Suk, N. Barov, J. B. Rosenzweig, and E. Esarey, Phys. Rev. Lett. 86, 1011 (2001).

[8] T. Tajima and J.M. Dawson, Phys. Rev. Lett. 43, 267 (1979).

[9] S. P. D. Mangles et al., Nature (London) 445, 741 (2004); C. G. R. Geddes et al., Nature (London) 431, 538 (2004); J. Faure et al., Nature (London) 431, 541 (2004).

[10] W. P. Leemans et al., Nature Phys. 2, 696 (2006).

[11] R. G. Hemker, N. M. Hafz, and M. Uesaka, Phys. Rev. ST Accel. Beams 5, 041301 (2002).

[12] A. Pukhov and J. Meyer-ter-Vehn, Appl. Phys. B 74, 355 (2002).

[13] K. Floetmann, http://desy.de/ mpyflo/.

[14] Chet Nieter and John R. Cary, J. Comput. Phys. 196, 448 (2004).

[15] Zhirong Huang and Kwang-Je Kim, Phys. Rev. ST Accel. Beams 10, 034801 (2007).

[16] A. Bacci, C. Maroli, V. Petrillo, A. R. Rossi, and L. Serafini, Study of the Beam Dynamics in a Linac with the Code RETAR PAC 2007 http://cern.ch/AccelConf/p07/ PAPERS/THPAN032.

[17] S. Reiche, Nucl. Instrum. Methods Phys. Res., Sect. A 429, 243 (1999); S. Reiche, http:// pbpl.physics.ucla.edu/ $\sim$ reiche. 